\title{
Episodic memory for object location versus episodic memory for object identity: Do they rely on distinct encoding processes?
}

\author{
STEFAN KÖHLER, MORRIS MOSCOVITCH, and BRENDA MELO \\ University of Toronto, Toronto, Ontario, Canada
}

\begin{abstract}
Three experiments were conducted to determine whether encoding processes that support episodic memory for object location are distinct from those that support memory for object identity. Guided by transfer-appropriate processing notions, we examined with an incidental learning paradigm whether an attentional focus on object location at encoding promotes subsequent recovery of object location, whereas a focus on object identity promotes recovery of object identity. We found that judging spatial relationships at encoding selectively supports recovery of object location, provided the test assesses memory for these relationships (rather than absolute location); our results also showed that judging physical and semantic object attributes promotes recovery of object identity preferentially. Contrasting with these domain-specific effects was evidence that identification processes involved in object naming boost memory for object identity as well as for absolute object location. Object identification at encoding may support memory performance in both domains by triggering the binding of identity and location information through a mechanism of object-based attentional selection.
\end{abstract}

The distinction between information about the identity of objects and about their spatial location has been of central importance to the cognitive neuroscience of visual perception and memory. In the realm of memory, evidence from functional neuroimaging suggests that the brain regions that support encoding and episodic retrieval of the spatial location of objects can be dissociated from those that support encoding and retrieval of object identity (Köhler, Moscovitch, Winocur, Houle, \& McIntosh, 1998; Moscovitch, Kapur, Köhler, \& Houle, 1995; Nyberg et al., 1996; Owen, Milner, Petrides, \& Evans, 1996). This line of research has also shown that not all brain regions show an involvement in memory processing that is specific to either information domain; some structures appear to support processes (encoding or retrieval) in both domains.

The present paper addresses, solely on the basis of behavioral data, whether episodic memory for object location and object identity relies on encoding processes that are domain specific - that is, that are distinct for each type of information. To investigate this issue we examined how

The experiments reported were part of S.K.'s dissertation, conducted in partial fulfillment of the requirements for the Ph.D. degree at the Department of Psychology, University of Toronto. We gratefully acknowledge the contributions of Gus Craik and Gordon Winocur as members of the dissertation committee. We also thank Gus Craik for his thoughtful comments on this manuscript and Julia Hong for her help with data collection. This research was supported by an NSERC Grant to M.M. S.K. was supported by an Ontario Graduate Scholarship. Correspondence should be addressed to S. Köhler, who is now at the Department of Psychology, University of Western Ontario, London, ON, N6A 5C2 Canada (e-mail: stefank@uwo.ca). different processing requirements at encoding affect memory recovery of the location as compared with the identity of visually apprehended objects.

Transfer-appropriate processing (TAP) notions provide a theoretical framework that is suitable to guide the examination of domain-specific encoding effects in memory processing. According to TAP, encoding conditions promote memory performance on later tests to the extent that the test permits transfer of the specific knowledge acquired at study (Blaxton, 1989; Morris, Bransford, \& Franks, 1977; Roediger, Weldon, \& Challis, 1989). For example, with respect to words, Morris et al. reported that attending to phonological attributes at encoding produces subsequently higher memory performance than attending to semantic attributes if the test used to measure performance requires retrieval of phonological information. Conversely, the authors showed that attending to semantic attributes of words at encoding produces higher memory performance on tests that are usually based on retrieval of semantic information (i.e., the typical levels-of-processing effect; Craik \& Lockhart, 1972). With respect to visually apprehended objects, one can ask whether an analogous relation holds for attending to their location and their identity: Do encoding tasks that require an attentional focus on the spatial location of objects support subsequent memory recovery of object location more than encoding tasks that require attending to the objects' identity? Further, does the opposite relation hold for memory recovery of object identity? Potential evidence demonstrating this pattern would suggest that retrieval of both types of information from episodic memory relies on domain-specific encoding processes. By contrast, the failure to detect such a pattern of performance could suggest that retrieval relies 
on common encoding processes that operate in both information domains.

Several studies have investigated the effects of encoding instructions on episodic memory for the location and the identity of visually presented objects (or pictures thereof). Generally, these studies have focused on the difference between two different types of intentional memory instructions (for review, see Ellis, 1990; Naveh-Benjamin, 1987). In one condition, participants are usually asked to prepare for a memory test on objects without being told that they will also be tested on spatial information. In the other one, they are asked to prepare for a memory test and specific reference is made to memory for object location. Although there are methodological problems associated with this approach (see below), some provocative evidence has been reported.

Pezdek and Evans (1979, Experiment 4) used a display with 16 photographs of named buildings that were placed in different locations on a map. Under spatial-encoding instructions, participants were asked to memorize the locations of the buildings; under object-encoding instructions, they were asked to memorize their visual appearance. Recognition memory performance for individual buildings was found to be higher after object encoding than after spatial encoding. In contrast, participants' accuracy in relocating the buildings on the map was statistically identical after both types of encoding instructions. Pezdek and Evans concluded from these results that recovery of object location from episodic memory does not rely on encoding processes that are distinct from those that support recovery of object identity. Other studies, however, have demonstrated sensitivity of memory for object location to encoding instructions in intentional learning paradigms. In these studies, conditions that emphasized the memorization of spatial information or that provided specific instructions on how to encode it produced better subsequent performance on memory tasks for object location than conditions that did not (e.g., Naveh-Benjamin, 1987, 1988; Park \& Mason, 1982).

A general criticism applying to intentional learning paradigms is that the processing strategies participants employ during learning are not under close control of the experimenter and may vary considerably across individuals. Most importantly, some but not all participants may deliberately use information on the spatial layout of displays as a mnemonic device to prepare for a memory test on the objects themselves (Mandler, Seegmiller, \& Day, 1977; Naveh-Benjamin, 1988). Given this problem, the described lack of consistency of findings is perhaps not so surprising.

Incidental learning paradigms, in which no reference is made to subsequent memory testing, offer a more promising method to test the domain specificity of encoding processes; they provide direct experimental control over the attentional focus and processing strategies participants apply to the stimulus material at encoding. We recently conducted a pilot experiment in the context of a functional neuroimaging study (Köhler et al., 1998), in which we used an incidental learning paradigm to examine en- coding effects on recognition memory for object location and object identity. The stimulus material consisted of a list of displays, each of which contained multiple line drawings of objects in distinct spatial configurations. At encoding, participants made same-different judgments on pairs of displays; matching had to be based either on the location or the identity of the objects presented. Participants' memory for the displays was tested with a forcedchoice recognition memory test that required the discrimination of targets from systematically altered novel test displays, which differed from targets in terms of either the location (spatial recognition test) or the identity (object recognition test) of one of the objects. Memory performance on the object recognition test was better after participants performed the object-matching task than after they performed the spatial-matching task. By contrast, on the spatial recognition task, performance levels after spatial matching did not exceed those after object matching. This pattern of results resembles that reported by Pezdek and Evans (1979) but is not subject to the criticisms that can be raised against their intentional learning paradigm. Thus, these results can also be considered as evidence that the encoding processes supporting episodic memory for object location are not distinct from those for object identity. It is noteworthy, however, that the matching tasks in the pilot experiment required only minimal processing of the displays; more demanding incidental encoding tasks may produce different effects.

The purpose of the present series of experiments was to elucidate more systematically, with an incidental learning paradigm, whether the encoding processes that support episodic memory for object location are distinct from those that support memory for object identity.

\section{EXPERIMENT 1}

In Experiment 1, we used the list of multiple-object displays from our pilot study and developed new incidental encoding tasks that required comparative judgments either on the identity or on the spatial relationship between the objects in these displays. At test, we employed a forced-choice recognition memory task and systematically varied the nature of the distractor (i.e., test display) to manipulate recovery of spatial and object information.

The literature on recognition memory for visual scenes suggests that, within the spatial domain, information about object location ${ }^{1}$ needs to be distinguished from information about spatial composition (Mandler \& Ritchey, 1977). Whereas object location specifies the association between a particular object and its location, spatial composition specifies areas of filled versus empty space in the scene, irrespective of the particular object occupying a filled space. In Experiment 1, we examined encoding effects on recognition memory for both types of spatial information.

Recognition memory for object identity was also investigated by focusing on two aspects separately-namely, memory for item information and memory for associative information (Murdock, 1974). Recognition memory for 
item information about object identity can be defined as familiarity with single objects irrespective of their associations with other objects in the same display. By contrast, recognition memory for associative information about object identity is thought to reflect recollection of associations between objects within a display.

\section{Method}

Overview and Design. The experimental design was a $3 \times 4$ mixed factorial with one factor between and one factor within participants. The between-participants manipulation was the type of task given at the time of encoding (intentional encoding, incidental object encoding, incidental spatial encoding). The within-participants manipulation was the type of memory decision required at recognition. Memory decisions varied with the type of test displays used to test recognition memory performance for different types of information.

Participants. Thirty-six undergraduate students at the University of Toronto participated in the experiment for credit in their introductory psychology course. Participants were assigned randomly to one of three encoding conditions and tested individually.

Materials. The stimuli consisted of representational line drawings of common objects taken from Snodgrass and Vanderwart's (1980) set of 260 normed pictures. Each display was composed of three drawings of objects that were not semantically related (Figure 1a). The pool of target displays consisted of 40 items. The drawings making up a display were presented at different locations on a 14-in. computer screen of a personal computer. To cover all possible locations, the area of the screen was divided into a $6 \times 8$ matrix, which was not visible during presentation of any of the items. All but the four corner cells of the matrix were designated as possible locations for the drawings. These 44 cells were used multiple times for the presentation of the entire list, but presentation frequencies were matched for all cells across the list. Every display contained drawings of unique objects presented in unique spatial conf igurations; the particular angles and distances between the three presentation locations of any target display were never used more than once in the same combination.

For memory testing, test displays were created that represented systematically altered target displays. For each target display, four different types of test displays were created. Spatial-novel displays were created in order to assess memory for spatial composition. They were constructed by moving one of the three objects to a novel location different from the one used in the corresponding target display, leading to novel unique spatial configurations (Figure 1b). Spatial-mixed displays were created to assess memory for object location. They were constructed by switching the position of two objects within the given configurations (Figure 1c). Object-novel displays were created to assess memory for item information about object identity. They were constructed by replacing one of the objects with a new object that had not been used in other displays. They could be distinguished from targets on the basis of familiarity with the identity of individual objects irrespective of their associated object context (Figure 1d). Object-mixed displays were created to assess memory for associative information about object identity. They were constructed by replacing one of the objects with a familiar object that had been used in another target display of the list (Figure 1e). Thus, these displays could not be distinguished from targets on the basis of familiarity with the identity of individual objects.

Procedure. In all encoding conditions, participants received practice before they were presented with the list of 40 target displays arranged in random order. Each display was shown for $5 \mathrm{sec}$. In the intentional-encoding condition, participants were asked to memorize the list of displays for a subsequent memory test. No specification was provided as to what aspects of the display participants should attend. The other two encoding conditions were incidental with no reference to a subsequent memory test. Judgments in both incidental encoding conditions were chosen to be comparative in order to match them in terms of relational processing demands. In the incidental object-encoding condition, participants were asked to rankorder the objects according to their subjective value and to indicate their answers by naming the objects from least valuable to most valuable. In the incidental spatial-encoding condition, participants made judgments about the spatial relationships between the objects' locations. For each display, they were asked to determine silently which object was in the middle horizontal position and then to judge which of the other two objects was closer to it (i.e., the object to the right or the one to the left). Participants indicated their answers by calling out "right" or "left."

Subsequent to the encoding phase, there was a delay of $30 \mathrm{~min}$ during which participants performed a distractor task unrelated to the memory test. After the delay, participants were administered a twoitem forced-choice recognition memory test. For every trial, a target display was paired with one of the four types of test displays. Each of the four test display types was used on 10 trials presented in random order across the entire set of 40 trials. Within a trial, both displays were presented in sequence for $3 \mathrm{sec}$ each. The target was presented first on half of the trials. Participants were asked to decide whether the first or the second display was one of the targets they had encountered before; they gave their responses by pressing one of two buttons of a response box. Participants could indicate their answers either while the second display of a pair was still present or during the following response interval of up to $10 \mathrm{sec}$. Participants had no knowledge about the composition of the list and the different types of test displays employed but were familiarized with the task on practice trials. Four different test lists with 40 trials were available for actual testing. Displays rotated across these four lists so that every target was combined with every test type once. The different lists were counterbalanced across participants in every encoding condition.

\section{Results}

Recognition memory performance is shown in Figure 2. An overall analysis of variance (ANOVA) was performed, which yielded a significant main effect of encoding task $\left[F(2,33)=32.34, M S_{\mathrm{e}}=0.02, p<.001\right]$. In addition, there was a significant interaction between encoding task and type of memory decision $\left[F(6,99)=3.62, M S_{\mathrm{e}}=0.01\right.$, $p=.003]$. The main effect of type of memory decision did not reach statistical significance $\left[F(3,99)=2.14, M S_{\mathrm{e}}=\right.$ $0.01, p=.101]$, indicating that all decisions were comparable in terms of their general task difficulty.

To examine the interaction between encoding task and type of memory decision, separate one-way ANOVAs were performed on each type of memory decision. Subsequently, Newman-Keuls tests were employed for post hoc comparisons. Encoding task had no significant effect on memory decisions involving spatial-novel displays $\left[F(2,33)=1.97, M S_{\mathrm{e}}=0.02, p=.846\right)$. In contrast, it did exert a significant effect on memory decisions involving spatial-mixed displays $\left[F(2,33)=11.30, M S_{\mathrm{e}}=0.01, p<\right.$ $.001]$, object-noveldisplays $\left[F(2,33)=29.72, M S_{\mathrm{e}}=0.02\right.$, $p<.001]$, and object-mixed displays $[F(2,33)=15.62$, $\left.M S_{\mathrm{e}}=0.02, p<.001\right]$. Post hoc comparisons revealed the same pattern for all memory decisions for which a significant effect was found; intentional encoding and incidental object encoding produced better memory performance than incidental spatial encoding (all $p s<.001$ ). When intentional encoding was compared with incidental object 
(a)

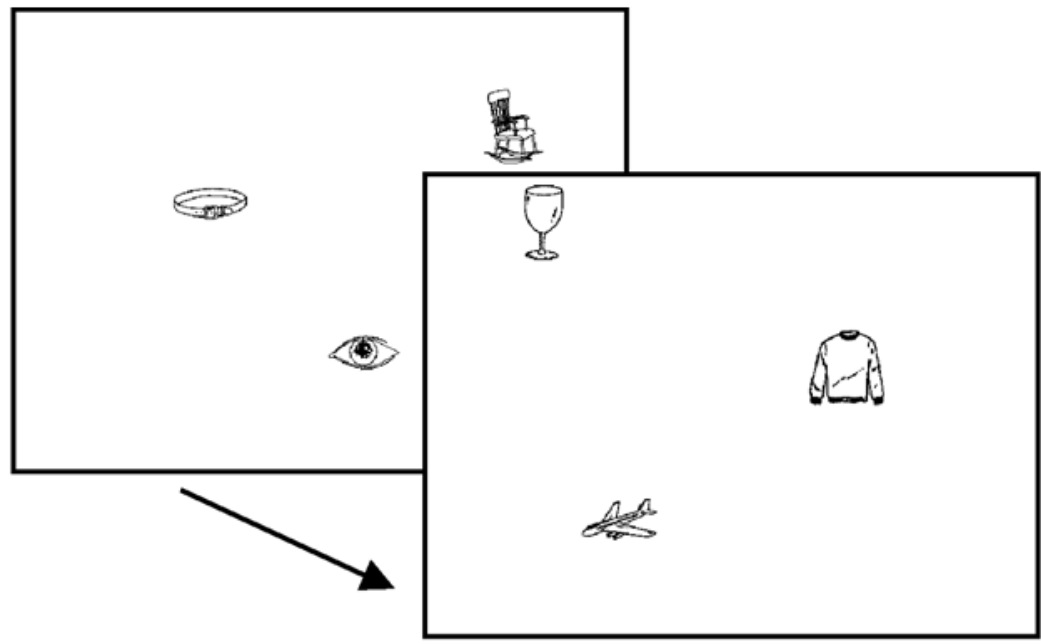

(b)

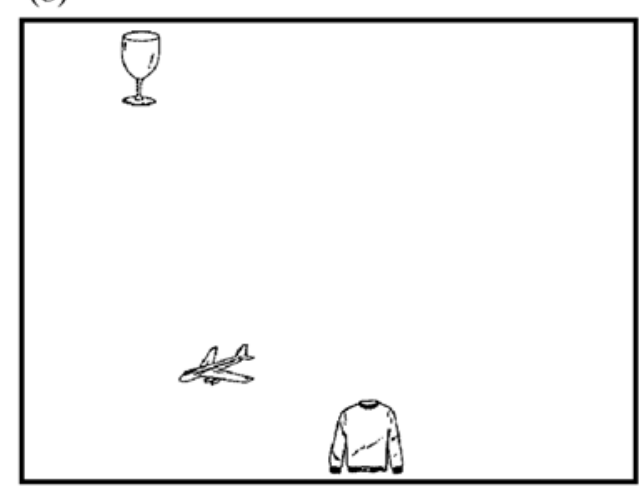

(d)

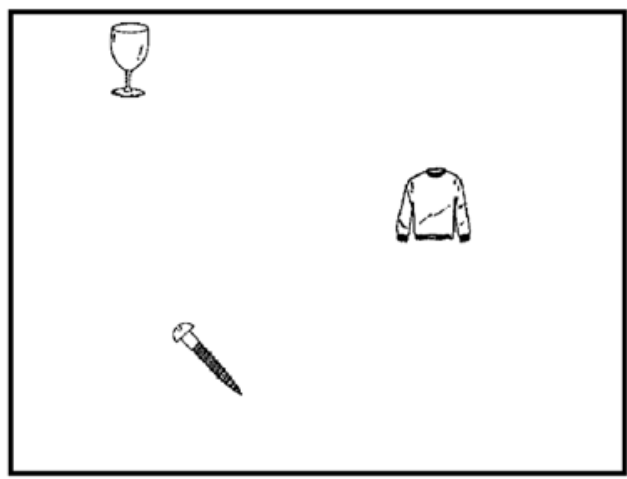

(c)

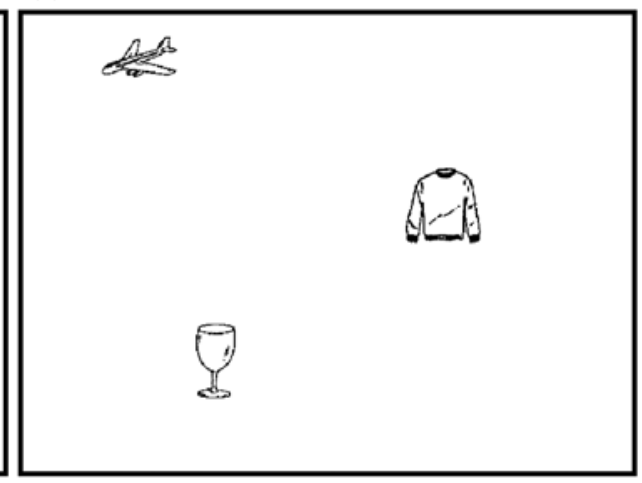

(e)

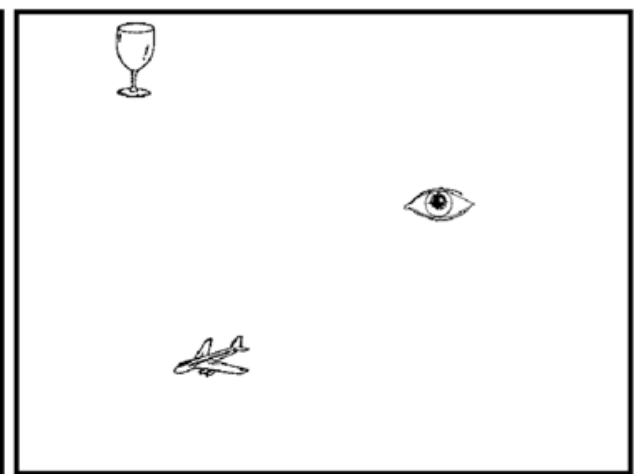

Figure 1. Display types used in Experiment 1. (a) Two of the target displays; (b) spatial-novel test display for the second item; (c) spatial-mixed test display; (d) object-novel test display; (e) object-mixed test display.

encoding, no significant differences were found for any type of memory decision (all $p \mathrm{~s}>.10$ ).

To examine whether participants retained any information about the displays after incidental spatial encoding, differences between the observed performance and the expected chance level (50\% correct decisions) were inspected using $t$ tests. Memory performance was significantly above chance only on memory decisions that re- quired retrieval of object location or spatial composition, which involved spatial-novel $[t(11)=3.86, p=.001]$ and spatial-mixed $[t(11)=2.35, p=.019]$ displays, respectively.

\section{Discussion}

The present experiment yielded two major results: First, when incidental encoding instructions were given that re- 
quired judgments on a semantic object attribute, performance on all memory decisions, those involving retrieval of associative and item-based object identity as well as those involving retrieval of object location and spatial composition, was high and indistinguishablefrom performance after intentional instructions. Second, subsequent to incidental encoding instructions that required judging the spatial relationships between object locations, performance was generally lower than in the other two encoding conditions, being above chance level only on those memory decisions that involved retrieval of spatial composition and object location.

That memory performance for associative and item information about object identity was high after semantic judgments relating to identity, but at chance after judgments on spatial relationships, can be interpreted as support for the operation of domain-specific encoding processes. The finding is in keeping with previous demonstrations that successful episodic recognition of object identity relies on meaning-based encoding when the memory test requires the discrimination of targets from test objects with a different name (Carroll, Byrne, \& Kirsner, 1985; Marks, 1991). To the extent that people exhibit a spontaneous tendency to process material in terms of its meaning when they are asked to memorize it (Craik \& Lockhart, 1972), our finding of equally high performance subsequent to intentional learning is consistent with this idea.

The evidence obtained with the test items for spatial information (object location and spatial composition) is more difficult to interpret in light of the notion of domainspecific encoding processes. Although judgments on spa- tial relationships between object locations at encoding did support recognition of object location and spatial composition at a level above chance, recognition performance did not exceed the level observed after semantic judgments relating to object identity. In fact, performance for object location was worse after spatial than after semantic judgments. A similar pattern of results was also observed in our pilot experiment, which was conducted with the same material but different incidental encoding tasks (Köhler et al., 1998). Thus, it seems unlikely that the present finding is related to the specific type of spatial judgment required during encoding. However, it is possible that memory for object location would benefit more from an attentional focus on spatial information if the encoding task involved more than one judgment of spatial attributes. This possibility was addressed in Experiment 2 .

To explain the observed pattern of results in its entirety, it may be necessary to perform a more refined examination of the match in processing requirements between the spatial encoding and retrieval tasks. Following TAP ideas, a spatial encoding task that requires attending to spatial relationships between objects may foster performance on a retrieval task for object location only to the extent that the test assesses memory for spatial relationships. For the recognition memory test in Experiment 1, it was impossible to determine whether participants based their memory decision on spatial relationships between objects or the absolute location of individual objects in relation to the boundaries of the displays. In Experiment 2, we modified the retrieval task in such a way that it allowed for the specific assessment of memory for spatial relationships.

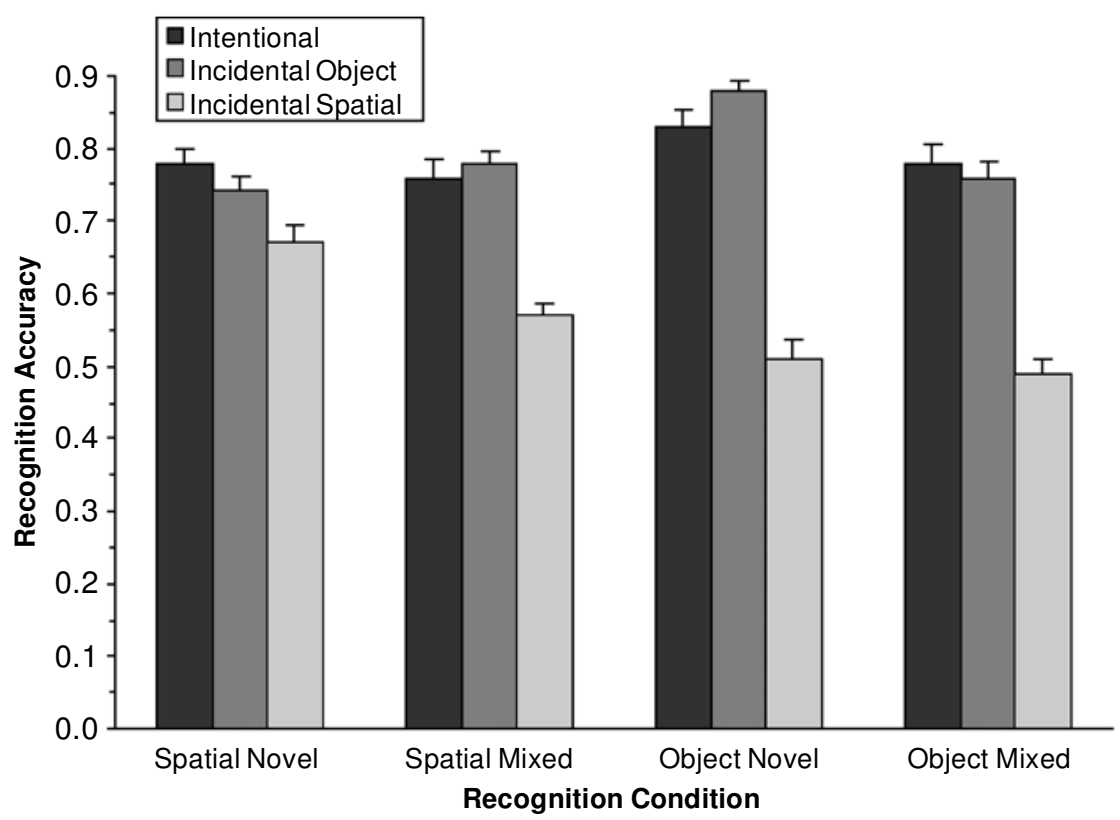

Figure 2. Mean proportional recognition accuracy as a function of type of encoding task and type of recognition condition. Error bars denote standard errors. 


\section{EXPERIMENT 2}

It is conceivable that memory performance for object location was so low after judgments on spatial relationships in Experiment 1 because the specific type of spatial processing required was insufficient to create a reliable memory representation of the locations in the three-object displays; a reliable representation may require processing of spatial attributes other than distances between object locations at encoding. To address this possibility, participants were asked to make an additional judgment on the angular relationships in the spatial-encoding condition of Experiment 2. To match general task requirements across conditions, a second judgment was also added to the object-encoding task.

A second goal of Experiment 2 was to determine whether attending to spatial relationships at encoding fosters memory for object location only to the extent that the location test assesses memory for spatial relationships. Naveh-Benjamin (1987) noted that information describing the spatial location of multiple objects in a display may be represented in memory in either of two reference frames. The location of a particular object may be represented in terms of its absolute location with the boundaries of the display being the reference frame; alternatively, it may be represented in terms of its location relative to that of the other objects providing the reference frame. Only the latter representation would code for the spatial relationships between object locations explicitly. To examine memory for relative and absolute object location separately, ${ }^{2}$ we employed a cued-recall test in Experiment 2 . This test allowed us to score participants' memory responses according to the two different spatial reference frames.

\section{Method}

Overview and Design. The experiment was based on a one-factor, between-participants design. The between-participants manipulation was the type of task administered at encoding and had four treatment levels: no encoding (chance baseline), combined encoding, object encoding, spatial encoding. The effects of this manipulation were examined on four different memory measures, which were obtained for every participant. Two measures tapped memory for object location and two tapped memory for object identity. Since these measures differed from each other qualitatively and different scales were used on different measures, they were examined separately in the statistical analyses.

Participants. Sixty-four University of Toronto undergraduate students participated in the experiment for credit in their introductory psychology course. Participants were tested individually and were assigned randomly to one of four experimental conditions.

Materials. Two lists of target displays were created and were used across participants in a counterbalanced order. Each list contained 12 unique displays that were composed in the same way as those in Experiment 1 (Figure 1a). A shorter list length was chosen because the memory tasks were expected to be more difficult than those in Experiment 1 . Each of the representational line drawings of objects selected for Experiment 2 had a name agreement score of more than 85\% (Snodgrass \& Vanderwart, 1980).

Displays were presented on sheets of $8.5 \times 11$ in. paper. As in the previous experiment, 44 locations were available for the presenta- tion of objects. These locations were neither marked on the sheets containing the target displays nor on those with the cue displays.

Each of the cue displays that was used for memory testing contained two of the three original drawings of objects presented in their original locations. The cue displays were created by removing the right-most object from four displays, the left-most object from four displays, and the middle object from four displays.

Procedure. All but the participants in the chance baseline condition went through an encoding phase first that required two judgments per display. After four trials with practice displays, they were presented with the 12 target displays arranged in random order. Each display was shown for a duration of $7 \mathrm{sec}$, during which time participants were asked to make two judgments; the order of these judgments was counterbalanced across participants in all conditions. In the spatial-encoding condition, participants made two judgments pertaining to the spatial relationships between object locations in each display. In addition to the distance judgment that was also part of Experiment 1, they judged the angular arrangement of the objects. For this judgment, participants first had to determine silently which object was in the middle position along the horizontal dimension of the sheet of paper. Subsequently, they had to judge whether the angle that was formed between the two imaginary lines connecting this object with the other two was smaller or larger than $90^{\circ}$. Participants indicated their answers by calling out "smaller" or "larger." In the object-encoding condition, participants made two judgments pertaining to object attributes. In addition to the value judgment that was also part of Experiment 1, they were required to rank-order the objects of each display according to the number of straight lines they contained. Participants indicated their answers by naming the objects from "drawn with the fewest straight lines" to "drawn with the most straight lines." Finally, in the combined encoding condition, participants made one judgment pertaining to object attributes of the display and one pertaining to spatial relationshipsthat is, the value judgment and the distance judgment from the other encoding conditions, respectively. No reference was made to a subsequent memory test in the encoding phase. Between encoding and memory testing, there was a delay of $5 \mathrm{~min}$ that was filled with unrelated distractor activity.

In the test phase, participants were presented with the 12 cues in random order. Before participants were tested on the target list, they were familiarized with the task on practice trials. For each of the cue displays, all participants who had completed the encoding phase were asked to make two memory judgments in succession. The order of these judgments was counterbalanced across participants.

To assess memory for object identity, participants were asked to select the target object that was missing in each cue display from a larger list of objects. This list was presented on a separate sheet of paper and contained the 12 missing objects (i.e., targets) from the study displays as well as 12 novel test objects (i.e., lures). Participants' memory performance for associative information about object identity was measured by determining the proportion of objects across all 12 trials that were correctly recognized as belonging to their corresponding cue display (correct associative recognition). To obtain a measure for the recognition of individual items, a familiarity error score was computed; it reflected the proportion of all errors made in which the incorrectly chosen object had been presented before, but not as part of the specific target display. A familiarity error was taken to indicate some effective encoding and retention of information about object identity in that it demonstrated familiarity with individual target objects. However, it reflected lack of access to information about the associated object context. By contrast, the selection of objects never presented was taken to indicate a complete lack of familiarity with the target objects.

In order to assess memory for object location, participants were asked to remember the location in which the missing objects of the cue displays had been presented during encoding. They were asked to mark this location on the sheet of paper containing the cue dis- 
play. Participants' memory performance for absolute object location was scored by measuring the Euclidian distance between the location indicated by the participant and the actual presentation location of the target object (i.e., mean displacement). This measure reflects memory for the location of the missing object in relation to the boundaries of the display; it does not take into account whether the remembered location is correct in terms of its spatial relationships to the other objects provided as cues. Memory for relative object location was evaluated by scoring the number of choices in which the remembered location was correct relative to the location of the other two objects in the horizontal and in the vertical dimension (i.e., correct relative locations). For example, the actual presentation location of a missing object may have been to the right of the other two objects in the horizontal dimension and between the two objects in the vertical dimension of the cue display. A choice was scored as correct only if it preserved both relationships for the remembered location; no credit was given for partially correct choices.

A no-encoding (chance baseline) condition was introduced in order to determine whether memory performance in the different encoding conditions was above the chance level. Chance level had to be determined empirically because not all measures allowed for its computation based on theoretically derived probabilities. Participants who were assigned to this condition underwent a test phase only. Rather than being asked to remember information from the study phase, they were simply instructed to complete the cue displays in such a way that they could be used in a memory experiment. Several constraints were provided, which reflected the rules that were followed by the experimenter for the generation of the displays. For each cue display, participants were asked to select a third object from the list that was developed for memory testing in the actual encoding conditions. Participants were also asked to provide a location for the third object.

\section{Results}

Memory performance for object identity is displayed in Table 1. The proportion of correctly recognized objects (correct associative recognition) indexes memory for associative information about object identity, whereas the proportion of familiarity errors indexes familiarity with individual objects irrespective of their associated object context. A one-way ANOVA yielded a significant effect of encoding task on the proportion of correctly recog-

Table 1

Means and Standard Deviations

for Performance on Memory Tests for Object Identity in Experiments 2 and 3

Performance Measure

\begin{tabular}{|c|c|c|c|c|}
\hline \multirow[b]{2}{*}{ Encoding Task } & \multicolumn{2}{|c|}{$\begin{array}{c}\text { Correct } \\
\text { Associative } \\
\text { Recognition* }\end{array}$} & \multicolumn{2}{|c|}{$\begin{array}{c}\text { Familiarity } \\
\text { Errors } \dagger\end{array}$} \\
\hline & $M$ & $S D$ & $M$ & $S D$ \\
\hline \multicolumn{5}{|l|}{ Experiment 2} \\
\hline Object & .30 & .21 & .86 & .15 \\
\hline Combined & .23 & .17 & .89 & .13 \\
\hline Spatial & .05 & .09 & .53 & .13 \\
\hline No encoding & .05 & .04 & .49 & .16 \\
\hline \multicolumn{5}{|l|}{ Experiment 3} \\
\hline Object & .30 & .13 & .96 & .07 \\
\hline Spatial with naming & .17 & .15 & .94 & .10 \\
\hline Spatial without naming & .08 & .07 & .58 & .16 \\
\hline No encoding & .03 & .05 & .48 & .12 \\
\hline
\end{tabular}

*Proportion of total responses. †Proportion of total errors. nized objects $\left[F(3,60)=12.99, M S_{\mathrm{e}}=0.02, p<.001\right]$. Newman-Keuls post hoc comparisons revealed significantly higher associative recognition memory performance after object $(p<.001)$ and combined encoding $(p<.001)$ than after spatial encoding. Performance levels after object and combined encoding did not differ significantly from each other $(p=.188)$ and were both above chance baseline $(p<.001$ and $p=.001$, respectively). In contrast, performance after spatial encoding was not significantly different from chance $(p=.838)$.

A one-way ANOVA also revealed a significant effect of encoding task for the proportion of familiarity errors to all errors made $\left[F(3,60)=34.80, M S_{\mathrm{e}}=0.02, p<.001\right]$. Newman-Keuls tests showed the same pattern of results as the corresponding tests for the average number of correctly recognized objects. Performance was better after object $(p<.001)$ and combined $(p<.001)$ than after spatial encoding. When participants committed errors, they predominantly selected previously presented objects (86\% and $89 \%$, respectively). In contrast, after spatial encoding, participants did not perform at a level different from chance baseline ( $p=.444)$, choosing as many objects that were previously presented to them $(53 \%)$ as novel ones $(47 \%)$. This response pattern indicates that participants in the spatial-encoding condition were not even familiar with individual target objects.

The data on memory for object location are presented in Table 2. Separate one-way ANOVAs were performed for both measures. The pattern of performance observed on the measure for absolute object location, mean displacement, was similar to the pattern observed on the memory measures for object identity. The corresponding ANOVA revealed a significant effect of encoding task $[F(3,60)=$ $\left.3.63, M S_{\mathrm{e}}=3.86, p=.018\right]$. Newman-Keuls tests indicated that performance after object encoding and combined encoding was significantly above the chance baseline ( $p=$ .040 and $p=.028$, respectively), whereas performance after spatial encoding did not differ significantly from chance $(p=.388)$.

Encoding task also exerted a significant effect on the measure for relative object location - that is, the number of locations correctly remembered in their relationship to the other objects $\left[F(3,60)=2.98, M S_{\mathrm{e}}=0.04, p=.038\right]$. Newman-Keuls tests revealed a pattern of results across encoding tasks that was different from the one for the absolute object location measure. Only those encoding tasks that included a spatial judgment tended to support memory for relative object location: Whereas there was a statistical trend for better-than-chance performance after combined $(p=.064)$ and spatial encoding $(p=.053)$, object encoding led to a performance level that was clearly not significantly different from chance $(p=.388)$.

\section{Discussion}

We found no evidence to support the hypothesis that an encoding condition that requires judgments of spatial relationships between object locations in multiple dimensions produces generally better performance in memory 
Table 2

Means and Standard Deviations

for Performance on Memory Tests

for Object Location in Experiments 2 and 3

\begin{tabular}{|c|c|c|c|c|}
\hline \multirow[b]{3}{*}{ Encoding Task } & \multicolumn{4}{|c|}{ Performance Measure } \\
\hline & \multicolumn{2}{|c|}{$\begin{array}{c}\text { Mean } \\
\text { Displacement* } \\
\end{array}$} & \multicolumn{2}{|c|}{$\begin{array}{c}\text { Correct } \\
\text { Relations } \dagger \\
\end{array}$} \\
\hline & $M$ & $S D$ & $M$ & $S D$ \\
\hline \multicolumn{5}{|l|}{ Experiment 2} \\
\hline Object & 6.26 & 1.79 & .50 & .19 \\
\hline Combined & 6.33 & 1.78 & .60 & .16 \\
\hline Spatial & 7.56 & 1.73 & .59 & .14 \\
\hline No encoding & 8.16 & 2.46 & .44 & .24 \\
\hline \multicolumn{5}{|l|}{ Experiment 3} \\
\hline Object & 5.40 & 1.28 & .63 & .14 \\
\hline Spatial with naming & 5.40 & 1.18 & .67 & .13 \\
\hline Spatial without naming & 7.25 & 2.28 & .58 & .18 \\
\hline No encoding & 8.29 & 2.22 & .45 & .19 \\
\hline
\end{tabular}

* Measured in centimeters. †Proportion of correct relative locations in horizontal and vertical dimensions.

for object location than a condition that requires no attentional focus on these spatial relationships. Instead, the comparison of memory for absolute and for relative object location suggested that the exact match in processing requirements between the spatial-encoding task and the memory test for object location is crucial. Consistent with our TAP-based predictions, we found that attending to spatial relationships at encoding promotes memory for object location only to the extent that the test assesses memory for spatial relationships between object locations. There was a statistical trend showing that memory for relative spatial location was supported by spatial encoding, whereas memory for absolute spatial location was clearly not significantly different from chance.

The pattern of results for the object-encoding condition, which required judgments on semantic and physical object attributes, was found to be opposite to that observed for spatial encoding when both spatial memory measures were examined; object encoding fostered memory for absolute but not for relative object location. Taken together, these results suggest that remembering the spatial relationships between object locations, but not the location of objects in absolute terms, relies on an attentional focus on the spatial relationships during encoding. The results obtained with the combined encoding task, which required judgments on the spatial relationships and on a semantic object attribute, are entirely consistent with this interpretation. Participants in this condition remembered as much information about absolute object location as did participants in the object-encoding condition and as much information about relative object location as did participants in the spatial-encoding condition.

It is important to note that the results for our measure for absolute object location paralleled those that were obtained with the memory measures for object identity; performance on all of these measures was better in the object-encoding and the combined encoding conditions than in the spatial-encoding condition. Given that only the former two conditions required naming of the objects at encoding, our findings could suggest that recovery of absolute object location, like object identity, relies on object identification processes involved in the naming response. In Experiment 3, further evidence was sought to address this hypothesis.

\section{EXPERIMENT 3}

The purpose of Experiment 3 was to examine further whether not only memory for object identity but also memory for absolute object location relies on the identification of the relevant objects at encoding. Chalfonte and Johnson (1996) noted that retention of information about object location requires binding of location information to information about the identity of the corresponding object. Recovery of absolute object location may depend on object identification at encoding because binding processes can take place only if the object's identity was determined in the first place. Binding may not be required for recovery of relative object location because information about spatial relationships can be represented in memory in terms of configurations that are independent of the identity of their elements.

To address the role of identification processes at encoding, we introduced a condition that was identical to the spatial-encoding condition in Experiment 2, except that it also required participants to name the objects; this condition was compared with the original spatial-encoding condition and the object-encoding condition of Experiment 2. On the basis of the considerations outlined above, spatial-encoding with object naming was expected to produce better memory performance for object identity and for absolute object location than spatial encoding without object naming. By contrast, memory for relative object location was expected to be unaffected by the naming manipulation.

\section{Method}

Overview and Design. The experiment was based on a onefactor between-participants design. The between-participants manipulation was the type of instructions given at the time of encoding. It had four treatment levels: no encoding (chance baseline), spatial encoding with naming, spatial encoding without naming, and object encoding. The effects of this manipulation were examined separately on the four memory measures that were used in Experiment 2.

Participants. Sixty-four University of Toronto undergraduate students participated in the experiment for credit in their introductory psychology course. Participants were tested individually and were assigned randomly to one of four experimental conditions.

Materials and Procedure. The stimulus material was the same as in Experiment 2. The encoding instructions given at the time of study were all incidental learning instructions. For two experimental conditions, they were the same as those given in Experiment 2: In the spatial encoding without naming condition, participants were instructed to make an angular judgment and a distance judgment without referring to the names of the objects. In the object-encoding condition, participants had to make a value judgment and a judgment that required them to rank-order the objects according to the number of straight lines they contained. The new encoding condition, spatial encoding with naming, required participants to make the same 
angular and distance judgments that participants made in spatial encoding without naming. However, participants were required to formulate their answers by making reference to the name of the objects. For the angular judgment, participants were required to indicate their answers by calling out sentences of the sort "the angle formed at $M$ (name of middle object) between $L$ (name of left object) and $R$ (name of right object) is smaller than 90 degrees." For the distance judgment, participants were asked to indicate their answers by calling out sentences of the sort " $L$ is closer to $M$ than $R$."

Participants were exposed to each display twice and were asked to provide their answers within the $4 \mathrm{sec}$ allotted for presentation. During each presentation, only one judgment was required. The second presentation of a display occurred only after the complete list of 12 displays had been shown. The order in which the two judgments were made at encoding was counterbalanced across participants.

All other aspects of the materials and the procedure were identical to those in Experiment 2.

\section{Results}

Memory performance for object identity is presented in Table 1. For the measure of associative recognition, a oneway ANOVA yielded a significant effect of encoding task $\left[F(3,60)=18.75, M S_{\mathrm{e}}=0.01, p<.001\right]$. Newman-Keuls comparisons indicated that associative recognition memory performance was above chance level after object encoding $(p<.001)$ and spatial encoding with naming $(p=$ .002) but did not differ significantly from chance baseline after spatial encoding without naming $(p=.218)$. Performance levels after object $(p<.001)$ and spatial encoding with naming ( $p=.024)$ were higher than after spatial encoding without naming. In addition, performance after object encoding was significantly higher than performance after spatial encoding with naming $(p=.001)$. Thus, the novel encoding condition of Experiment 3, spatial encoding with naming, produced a level of performance that was between the one observed after object encoding and that after spatial encoding without naming.

For the proportion of familiarity errors to all errors committed, a one-way ANOVA revealed a significant effect of encoding task as well $\left[F(3,60)=69.56, M S_{\mathrm{e}}=\right.$ $0.01, p<.001]$. Newman-Keuls tests showed that performance was better after object encoding $(p<.001)$ and spatial encoding with naming $(p<.001)$ than after spatial encoding without naming, but not significantly different between the former two conditions $(p=.730)$. In both conditions, when participants committed errors, they predominantly selected previously encountered (96\% and 94\%, respectively) objects. Spatial encoding without naming produced a performance level that was above chance baseline ( $p=.017)$ but low; participants chose only slightly more previously presented objects $(58 \%)$ than novel ones $(42 \%)$.

Table 2 displays the data on memory for object location. Separate one-way ANOVAs were performed for both measures. Encoding task had a significant effect on the measure for absolute object location, mean displacement $\left[F(3,60)=9.98, M S_{\mathrm{e}}=3.28, p<.001\right]$. Newman-Keuls comparisons indicated that performance after object encoding and after spatial encoding with naming did not dif- fer from each other and was above chance (for both, $p<$ $.001)$, whereas performance after spatial encoding without naming did not exceed the chance baseline $(p=.464)$. Importantly, spatial encoding with naming produced better performance on mean displacement scores than spatial encoding without naming $(p=.028)$.

Data for the memory measure of relative object location showed a different pattern across encoding conditions $\left[F(3,60)=5.42, M S_{\mathrm{e}}=0.03, p=.002\right.$; see Table 2]. Post hoc comparisons showed that all encoding tasks promoted memory for relative object location to some extent in that they produced above-chance performance levels (spatial encoding without naming, $p=.026$; spatial encoding with naming, $p=.002$; object encoding, $p=$ .008). However, the corresponding performance scores did not differ from each other (all $p \mathrm{~s}>.10$ ).

\section{Discussion}

The results obtained in Experiment 3 support the idea that memory for the identity of objects and their absolute location is dependent on processes of object identification at encoding: Judgments on spatial relationships at encoding produced a higher level of subsequent memory performance when they required naming of the objects than when they did not. This effect was most pronounced on the measure for associative recognition and the measure for absolute location. On both measures, performance was at chance in the spatial-encoding condition that did not require object naming but clearly exceeded the chance level in the condition that did. As noted earlier, this pattern of results can be explained, even for the absolute-location measure, if the role of binding processes is taken into consideration. We shall elaborate on this issue in the General Discussion section.

In keeping with our predictions, we found a pattern of performance for memory for relative object location that differed from the one observed for absolute object location. Performance was unaffected by the naming manipulation and above chance level in both spatial-encoding conditions, suggesting that recovery of spatial relationships between object locations does not rely on processes of object identification involved in naming at encoding. The findings from Experiment 2 indicated that recovery of spatial relationships may, instead, require that participants direct their attention to these spatial relationships at encoding. However, in Experiment 3, performance on the measure for relative object location was as good in the objectencoding condition as in the two spatial-encoding conditions, providing no further support for this hypothesis.

It is worth noting that when participants named the objects in the context of judgments about physical and semantic object attributes, performance on the associative recognition score for object identity was even better than when they named the objects in the context of spatial judgments. By contrast, memory performance for neither absolute nor relative object location showed this improvement. Inasmuch as the observed memory benefits resulted 
from judging specific physical and semantic object attributes, these results can be interpreted as an encoding effect that is specific to the object domain.

\section{GENERAL DISCUSSION}

The present experiments were conducted to determine whether the encoding processes that support episodic memory for object location are distinct from those that support memory for object identity. Guided by general ideas adapted from the TAP framework, we hypothesized that incidental encoding tasks that orient participants' attention to the location of objects preferentially support retrieval on memory tests for object location, whereas tasks that orient attention to semantic or physical attributes of the objects preferentially support retrieval on tests for object identity. Although there was evidence in each of the three experiments that pointed to the operation of distinct encoding processes, our results clearly show that not all encoding processes are distinct for the two domains - that is, are domain-specific. Most importantly, we found that processes related to object naming support memory performance across domains. We shall summarize and discuss the evidence in support of distinct and common encoding processes in turn.

\section{Evidence for Distinct Encoding Processes}

Two sources of evidence in our data can be interpreted as support for the operation of domain-specific encoding processes. First, encoding judgments on semantic object attributes alone (Experiment 1) or on semantic and physical attributes together (Experiments 2 and 3 ) produced better performance on memory tests for object identity than did encoding judgments on the objects' spatial relationships. This pattern held for measures of item and associative information about object identity provided the spatial judgments did not require naming of the objects whose relationships were being judged. However, even when spatial judgments did require a naming response, judging semantic and physical object attributes produced higher performance on the recognition measure for associative object information (Experiment 3). These data hint that attending to specific object attributes, over and above those that are essential for object naming, can have domain-specific memory effects and support recovery of object identity preferentially (for a discussion of the role of naming, see below).

Second, our results showed that judging spatial relationships between object locations at encoding consistently supported subsequent memory retrieval of information about object location but not about object identity. Experiments 2 and 3 showed that it was a particular type of spatial information that could be retrieved successfully subsequent to making these judgments at encoding, namely information about the spatial relationships between objects (relative object location). To the extent that memory recovery of relative object location can rely on spatial encoding processes that do not promote memory recovery of object identity, these results can be taken as support for the presence of domain-specific encoding processes. However, only in Experiment 2 did we find evidence that spatial judgments at encoding produced better performance on a memory test for relative object location than encoding judgments relating to object identity. In Experiment 3, memory performance was comparable in both encoding conditions. This pattern of results suggests that, even though there are encoding processes that promote recovery of spatial relationships selectively, these processes do not necessarily operate more effectively than common encoding processes that foster memory performance across domains.

\section{Evidence for Common Encoding Processes}

We found that encoding judgments on attributes relating to object identity supported performance not only on memory tests for the identity of these objects but also on tests for their location. This pattern of results was found consistently across different types of retrieval tasks-that is, irrespective of whether the task involved recognition (Experiment 1) or cued recall (Experiments 2 and 3 ) of object location. Most notably, when the test assessed recovery of absolute object location, judgments on object attributes produced a performance level that was not inferior but that was equal or even superior to the one resulting from judgments on spatial relationships (Experiments 2 and 3).

The observed absence of benefits from attending to spatial relationships for recovery of absolute location parallels findings from previous experiments with intentional learning paradigms, in which participants' efforts to memorize object location failed to improve location-memory performance (Ellis, 1990; Mandler et al., 1977; Pezdek \& Evans, 1979; von Wright, Gebhard, \& Kartunnen, 1975; Zechmeister, McKillip, Pasko, \& Bespalec, 1975). These findings led to the proposal that encoding of information about the location of objects proceeds automatically and does not require the allocation of attentional resources (Hasher \& Zacks, 1979). However, experiments based on intentional learning paradigms do not allow for an identification of the specific encoding processes that are critical for subsequent successful remembering in either domain.

The results of the present experiments, obtained with an incidental encoding paradigm, shed some light on the nature of the common processes that are effective at encoding in both domains; they point to the importance of processes related to object identification. Across experiments, only encoding conditions that required the identification of the displayed objects for a naming response produced a high level of familiarity with the objects' identity together with good relocation accuracy in terms of absolute location. Experiment 3 showed that even judgments on spatial relationships between objects can produce a high level of familiarity with the objects' identity, together with good absolute relocation accuracy, provided they require the identification of the relevant objects.

Previous research suggests that object identification processes at encoding can promote subsequent memory retrieval even when they do not result in an overt naming 
response (Madigan, 1983; Nelson, 1979). For example, judging objects in terms of a semantic attribute promotes performance on recognition memory tests for object identity and on relocation measures even when no naming response is required at encoding (Carroll et al., 1985; Mandler et al., 1977). There is also evidence showing that people are able to remember the identity and absolute location of pictures of meaningless, abstract objects that cannot easily be named (e.g., Nunn, Polkey, \& Morris, 1998). Thus, it is possible that encoding processes involved in establishing a perceptual representation of the structural description of an object (Riddoch \& Humphreys, 1987) are sufficient to allow for subsequent recovery of its identity and absolute location from episodic memory. At present, however, this idea remains a speculation. Further research is necessary to determine with certainty the extent to which object identification processes other than those involved in naming promote memory across domains and the extent to which the outcome depends on the specific processing demands of the retrieval task used. ${ }^{3}$

\section{The Role of Binding and Object-Based Attentional Selection in Encoding Object Location}

Why are object identification processes involved in a naming response critical not only for subsequent recovery of object identity but also for recovery of absolute object location? To answer this question, it is important to note that in all three experiments reported the memory test for location information required recovery of the location of particular objects. Chalfonte and Johnson (1996) argued that establishing memory representations for the location of specific objects requires that location information is bound to information about the identity of the corresponding objects. Following this idea, memory retrieval of the absolute location of specific objects may rely on their identification at encoding because this information can be subsequently recovered only if it was tagged to corresponding information about object identity. By contrast, recovery of relative object location, which was found to be independent of object identification, may not rely on these cross-domain binding processes because spatial relationships can be represented in memory as distinct configurations irrespective of the identity of their elements.

Once the role of identification and binding processes is acknowledged, there is a further issue that requires consideration: Why does binding of absolute object location and object identity not require that participants' attentional focus be oriented to location information at encoding? Why should processes of object identification be sufficient to support subsequent memory performance? Although a comprehensive discussion of this issue is beyond the scope of the present paper, it is noteworthy that our results can be accommodated if one considers that entire objects can be the units of attentional selection (e.g., O'Craven, Downing, \& Kanwisher, 1999). Treisman (1998) proposed that when an entire object is selected, the activated perceptual representation includes all of its perceived attributes, including its present location. If one assumes that object naming evokes an attentional focus on entire objects, then it becomes explicable why absolute location can be recovered even when the encoding task does not require any focus on spatial information. This account can also help to clarify why the intention to memorize object location is not a prerequisite for successful location memory performance in intentional learning paradigms; the intention to memorize an object's identity may already trigger attentional selection of the entire object, including its absolute location, and thus allow for the execution of cross-domain binding processes.

In sum, the present findings show that successful recovery of object location and object identity from episodic memory relies on an intricate interplay between domainspecific and common encoding processes. The reported behavioral evidence complements findings from functional neuroimaging, which indicates that encoding processes for object location and object identity have common as well as distinct neural correlates (Köhler et al., 1998; Moscovitch et al., 1995; Nyberg et al., 1996; Owen et al., 1996). Our data suggest that attending to spatial relationships between objects at encoding involves processes that selectively support recovery of object location, provided the test assesses memory for spatial relationships between these locations. By contrast, judging objects in terms of specific physical or semantic attributes invokes processes that can promote recovery of object identity selectively. Our findings indicate that identification processes involved in object naming play a common role in encoding; they contribute to producing a sense of familiarity with the objects encountered and to establishing in memory their absolute location. We propose that object identification processes support memory performance in both domains by triggering the binding of identity and absolute location information through a mechanism of objectbased attentional selection.

\section{REFERENCES}

Blaxton, T. A. (1989). Investigating dissociations among memory measures: Support for a transfer-appropriate processing framework. Journal of Experimental Psychology: Learning, Memory, \& Cognition, 15, 657-668.

Carroll, M., Byrne, B., \& Kirsner, K. (1985). Autobiographical memory and perceptual learning: A developmental study using picture recognition, naming latency, and perceptual identification. Memory \& Cognition, 13, 273-279.

Chalfonte, B. L., \& Johnson, M. K. (1996). Feature memory and binding in young and older adults. Memory \& Cognition, 24, 403-416.

Craik, F. I. M., \& Lockhart, R. S. (1972). Levels of processing: A framework for memory research. Journal of Verbal Learning \& Verbal Behavior, 11, 671-684.

ELLIS, N. R. (1990). Is memory for spatial location automatically encoded? Memory \& Cognition, 18, 584-592.

HASHER, L., \& ZACKS, R. T. (1979). Automatic and effortful processes in memory. Journal of Experimental Psychology: General, 108, 356-388.

Köhler, S., Moscovitch, M., Winocur, G., Houle, S., \& McIntosh, A. R. (1998). Networks of domain-specific and general regions involved in episodic memory for spatial location and object identity. Neuropsychologia, 36, 129-142. 
Madigan, S. (1983). Picture memory. In J. C. Yuille (Ed.), Imagery, memory, and cognition: Essays in honor of Allan Paivio (pp. 65-89). Hillsdale, NJ: Erlbaum.

MandleR, J. M., \& Ritchey, G. H. (1977). Long-term memory for pictures. Journal of Experimental Psychology: Human Learning \& Memory, 3, 386-396.

Mandler,J. M., Seegmiller,D., \& Day, J. (1977). On the coding of spatial information. Memory \& Cognition, 5, 10-16.

MARKs, W. (1991). Effects of encoding the perceptual features of pictures on memory. Journal of Experimental Psychology: Learning, Memory, \& Cognition, 17, 566-577.

Morris, C., BRANSFord, J., \& Franks, J. (1977). Levels of processing versus transfer appropriate processing. Journal of Verbal Learning \& Verbal Behavior, 16, 519-533.

Moscovitch, M., Kapur, S., Köhler, S., \& Houle, S. (1995). Distinct neural correlates of visual long-term memory for spatial location and object identity: A positron emission tomography study in humans. Proceedings of the National Academy of Sciences, 92, 3721-3725.

Murdock, B. B., JR. (1974). Human memory: Theory and data. Potomac, MD: Erlbaum.

Naveh-Benjamin, M. (1987). Coding of spatial location information: An automatic process? Journal of Experimental Psychology: Learning, Memory, \& Cognition, 13, 595-605.

NaveH-Benjamin, M. (1988). Recognition memory of spatial location information: Another failure to support automaticity. Memory \& Cognition, 16, 437-445.

NeLSON, D. L. (1979). Remembering pictures and words: Appearance, significance, and name. In L. S. Cermak \& F. I. M. Craik (Eds.), Levels of processing in human memory (pp. 45-76). Hillsdale, NJ: Erlbaum.

Nunn, J. A., Polkey, C. E., \& Morris, R. G. (1998). Selective spatial memory impairment after right unilateral temporal lobectomy. $\mathrm{Neu}$ ropsychologia, 36, 837-848.

Nyberg, L., McIntosh, A. R., Cabeza, R., Habib, R, Houle, S., \& TulVING, E (1996). General and specific brain regions involved in encoding and retrieval of events: What, where, and when. Proceedings of the National Academy of Sciences, 93, 11280-11285.

O'Craven, K. M., Downing, P. E., \& Kanwisher, N. (1999). fMRI evidence for objects as the units of attentional selection. Nature, 401, 584-587.

Owen, A. M., Milner, B., Petrides, M., \& Evans, A. C. (1996). Memory for object features versus memory for object locations: A positron emission tomography study of encoding and retrieval processes. Proceedings of the National Academy of Sciences, 93, 9212-9217.

PARK, D. C., \& MASON, D. A. (1982). Is there evidence for automatic processing of spatial and color attributes present in pictures and words? Memory \& Cognition, 10, 76-81.
Pezdek, K., \& Evans, G. W. (1979). Visual and verbal memory for objects and their spatial locations. Journal of Experimental Psychology: Human Learning \& Memory, 5, 360-373.

RidDoch, M. J., \& Humphreys, G. W. (1987). Visual object processing in optic aphasia: A case of semantic access agnosia. Cognitive Neuropsychology, 4, 131-185.

Roediger, H. L., III, Weldon, M. S., \& Challis, B. H. (1989). Explaining dissociations between implicit and explicit measures of retention: A processing account. In H. L. Roediger III \& F. I. M. Craik (Eds.), Varieties of memory and consciousness: Essays in honour of Endel Tulving (pp. 3-41). Hillsdale, NJ: Erlbaum.

SNOdgrass, J. C., \& VANDERWART, M. (1980). A standardized set of 260 pictures: Norms for name agreement, image agreement, familiarity, and visual complexity. Journal of Experimental Psychology: Human Learning \& Memory, 6, 174-215.

Treisman, A. (1998). The perception of features and objects. In R. D. Wright (Ed.), Visual attention (pp. 26-54). New York: Oxford University Press.

von Wright, J. M., Gebhard, P., \& Kartunnen, M. (1975). A developmental study of the recall of spatial location. Journal of Experimental Child Psychology, 20, 181-190.

Zechmeister, E. B., McKillip, J., Pasko, S., \& Bespalec, D. (1975). Visual memory for place on the page. Journal of General Psychology, 92, 43-52.

\section{NOTES}

1. Mandler and Ritchey (1977) called this information "spatial location." We replace it with the term object location to indicate that the locations of relevance here are all occupied by distinct objects.

2. Of course, information specifying absolute object location is not completely independent from information specifying relative object location. A participant scoring perfect on a measure for absolute object location will also score perfect on a measure for relative object location. Nevertheless, at a lower performance level, the two measures can convey partially independent information.

3 . There is evidence to suggest, for example, that recognition memory for the identity of objects relies less on object identification at encoding if the memory test requires the discrimination of targets from lures with the same name but different perceptual attributes (Marks, 1991).

(Manuscript received November 15, 1999; revision accepted for publication July 4, 2001.) 